\title{
Tetrabromobisphenol A recovery from computer housing plastic by a new solvothermal process
}

\author{
Cong-Cong Zhang • Veena Sahajwalla • \\ Fu-Shen Zhang
}

Received: 21 September 2013/Accepted: 3 January 2014/Published online: 24 January 2014

(C) Springer International Publishing Switzerland 2014

\begin{abstract}
Brominated flame retardants contained in electrical and electronic waste plastic are toxic to both humans and the environment. Most disposal technologies for brominated flame retardants are environmentally unfriendly. Here, a novel solvothermal process was designed to recover tetrabromobisphenol A, a typical brominated flame retardant, from waste computer housing plastic. The plastic waste was treated by the solvothermal process followed by vacuum rotary evaporation. Results show a tetrabromobisphenol A recovery efficiency of $78.9 \%$, and a purity of $95.6 \%$. The stability of tetrabromobisphenol A during the solvothermal process was confirmed by nuclear magnetic resonance. Kinetics showed that diffusion across the polymer layer controlled recovery. We conclude that the novel solvothermal process is a promising green way to recover tetrabromobisphenol A from electrical and electronic waste plastic.
\end{abstract}

Keywords Electrical and electronic waste - Solvothermal technique - Brominated flame retardant .

Tetrabromobisphenol A - Chemical recycling - Solvent extraction

C.-C. Zhang · F.-S. Zhang ( $₫)$

Research Center for Eco-Environmental Sciences, Chinese Academy of Sciences, 18 Shuangqing Road, Beijing 100085, China

e-mail: fszhang@rcees.ac.cn

V. Sahajwalla

Centre of Sustainable Materials and Research Technology,

University of New South Wales, Sydney, NSW 2052, Australia

\section{Introduction}

Tetrabromobisphenol A, which holds about $50 \%$ of the market in brominated flame retardants (Liu et al. 2006), are widely used as an additive flame retardant in plastic to increase the fire resistance of the electrical and electronic equipment (Alaee et al. 2003; Schlummer et al. 2007). With the rapid growth of electronic waste during the last two decades (Heart 2008), large quantities of tetrabromobisphenol A containing plastic are generated worldwide every year (Vilaplana et al. 2009). Tetrabromobisphenol A has been detected in various environmental matrices, and they negatively affect various aspects of mammalian and human physiology (Luo et al. 2011). Treatment of tetrabromobisphenol A containing plastic has become a challenging task especially due to these harmful effects of tetrabromobisphenol A on both humans and environment.

The majority of these tetrabromobisphenol A containing plastic are currently either dumped in landfills (Osako et al. 2004) or incinerated without energy recovery (Sakai et al. 2001). Nevertheless, neither of these two treatments are environmental benign approaches due to the production of secondary environmental pollution such as polybrominated dibenzo- $p$-dioxins/furans caused by tetrabromobisphenol A (Osako et al. 2004; Sakai et al. 2001). Tetrabromobisphenol A removal and recovery from waste plastic is urgently desired to reduce such negative environmental effects of tetrabromobisphenol $\mathrm{A}$ as well as recycle this type of valuable resources, which also meets the requirement of European Union's Directive on waste electrical and electronic equipment (Imai et al. 2003).

Conventional removal of tetrabromobisphenol A from plastic is usually performed by Soxhlet extraction, which requires long extraction time and high solvent consumption (Heart 2008). Various advanced solvent-based recycling 
technologies, including supercritical fluid extraction (Gamse et al. 2000; Wang et al. 2004), pressurized liquid extraction (Schlummer et al. 2005), ultrasonic-assisted extraction (Vilaplana et al. 2008) and microwave-assisted extraction ( $\mathrm{Li}$ et al. 2009), have also been extensively studied. However, these technologies mean high energy cost and equipment requirement, which have become challenges faced by them to recycle tetrabromobisphenol A from plastic (Guo et al. 2009). In our previous work, we reported that solvothermal process conducted at $90{ }^{\circ} \mathrm{C}$ holding for $120 \mathrm{~min}$ with the liquid to solid ratio of $15: 1$ using methanol as solvent was a powerful method to remove brominated flame retardant from electrical and electronic waste plastic (Zhang and Zhang 2012). As a consequence, we applied this technique followed by rotary evaporation treatment to recycle tetrabromobisphenol A from computer housing plastic. Thus far, less report is available on the recovery of tetrabromobisphenol A from computer housing plastic by solvothermal process. The aim of the present study was to demonstrate the feasibility of solvothermal process to recycle tetrabromobisphenol A from computer housing plastic. The quality of the recycled tetrabromobisphenol A was evaluated, and the rate controlling step of solvothermal process was identified.

\section{Experimental}

\section{Materials}

Waste computer housing plastic was supplied by XIAMEN OASIS Sources Corporation Limited. It was identified as acrylonitrile-butadiene-styrene resin with tetrabromobisphenol $\mathrm{A}$ as additive flame retardant. The plastic was ground to lower than $1 \mathrm{~mm}$ by a grinder under cooling condition by liquid nitrogen, and particle size of 14-20 mesh was used. Acetone and methanol were of pesticide analysis grade (J\&K Chemical Limited, USA). Tetrabromobisphenol A technical grade was supplied by ACROS Organics, USA. Anhydrous sodium sulfate was purchased from Sinopharm Chemical Reagent Corporation Limited (Beijing, China).

\section{Experimental procedures}

Solvothermal treatment was carried out as described in a previous study (Zhang and Zhang 2012), indicating that the optimum temperature, time and liquid to solid ratio for solvothermal treatment to remove tetrabromobisphenol A were $90{ }^{\circ} \mathrm{C}, 120 \mathrm{~min}$ and $15: 1$, respectively. Solvothermal treatment conducted under these conditions using methanol as solvent was thus repeated multiple times. The solid and solvent mixture were removed from the reactor and separated by centrifugation $(10,000 \mathrm{~g}, 10 \mathrm{~min})$. Then, the solvent phases were collected and evaporated on the vacuum rotary evaporator to obtain recycled tetrabromobisphenol A powder. Different treatment times $(15,30,45$, $60,75,90,105,120,180$ and $240 \mathrm{~min}$ ) were varied to optimize the tetrabromobisphenol A recovery efficiency of solvothermal process.

Analytical procedures

Tetrabromobisphenol A was determined by gas chromatography-mass analysis of tetrabromobisphenol $\mathrm{A}$ in the solvent phase, which was accomplished by an Agilent 7890A gas chromatograph equipped with an Agilent 5975C mass spectrometry detector (USA). The purity of tetrabromobisphenol A was calculated by comparing the peak areas of the gas chromatography-mass profiles for the recycled tetrabromobisphenol $\mathrm{A}$ and tetrabromobisphenol A standard material. The structure of recycled tetrabromobisphenol A powder was examined by analysis of nuclear magnetic resonance. The ${ }^{13} \mathrm{C}$ and ${ }^{1} \mathrm{H}$ nuclear magnetic resonance spectra of tetrabromobisphenol A dissolved in methanol- $\mathrm{d}_{4}$ were obtained at room temperature on a JEOL $600 \mathrm{MHz}$ spectrometer (Tokyo, Japan). All the experiments were carried out in triplicate for parallel test, and only the average values with standard errors were reported. Blank levels for the solvothermal procedure were typically lower than $1 \%$ of tetrabromobisphenol A content in waste computer housing plastic.

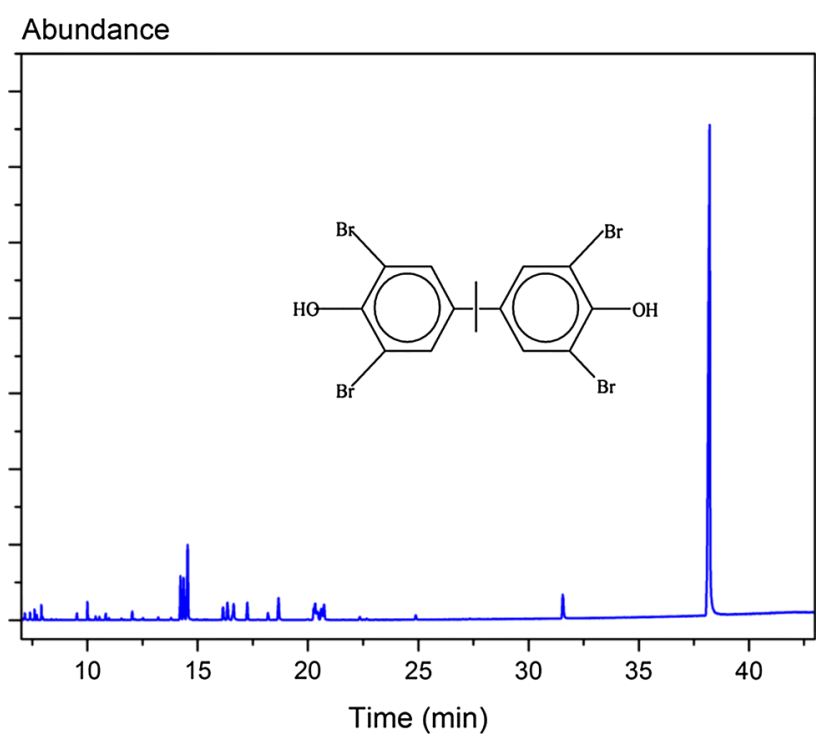

Fig. 1 Gas chromatography-mass chromatogram of the solvothermal extract. Conditions: solvent methanol; temperature $90{ }^{\circ} \mathrm{C}$; time $120 \mathrm{~min}$; liquid to solid ratio 15:1. Note that tetrabromobisphenol A is the major component of the extract, which can be recycled by following vacuum rotary evaporating treatment 


\section{Results and discussion}

\section{Recovery efficiency of tetrabromobisphenol A}

The gas chromatography-mass chromatogram of solvent phase after solvothermal treatment shows that tetrabromobisphenol $\mathrm{A}$ is the major component of the extract (Fig. 1). The observed small quantity of impurities is attributed to the thermal decomposition of acrylonitrilebutadiene-styrene resin (Wang et al. 2004), and the purity of tetrabromobisphenol A could be further improved by recrystallization purification. Figure $2 \mathrm{a}$ implies that tetrabromobisphenol A recovery increased as the prolonging of time till about $120 \mathrm{~min}$ and then remained basically unchanged. According to a previous study (Zhang and Zhang 2012), the residue tetrabromobisphenol A was mainly remained in the solid phase, which could be recovered by further solvothermal treatment. Tetrabromobisphenol A recovery efficiency achieved highest of $78.9 \%$ after 120 min treatment. As chemical feedstocks, the quality of the recycled tetrabromobisphenol A was also of great significance.
Quality of the recycled tetrabromobisphenol A

After the solvent phases were collected and evaporated to dryness, slight yellow tetrabromobisphenol A powder with a purity of $95.6 \%$ was obtained (Fig. 2b), indicating that tetrabromobisphenol A in the extract was not absolutely pure due to the dissolution of polymeric materials after solvothermal treatment (Altwaiq et al. 2003). As shown in ${ }^{13} \mathrm{C}$ nuclear magnetic resonance spectra of the recycled tetrabromobisphenol A and pure tetrabromobisphenol A (Fig. 3a), the peak with $\delta_{\mathrm{C}}$ of $49.15 \mathrm{ppm}$ is related with the carbon of methanol- $\mathrm{d}_{4}$; the relative high chemical shift at $149.13 \mathrm{ppm}$ is ascribed to the $\mathrm{C}(f, i)$; the signal at $143.95 \mathrm{ppm}$ is assigned to the $\mathrm{C}(c, l)$; the peak at $130.36 \mathrm{ppm}$ corresponds to the $\mathrm{C}$ $(b, d, g, k)$; the peak at $110.87 \mathrm{ppm}$ is ascribed to the $\mathrm{C}(a, e$, $h, j)$; the peak at 41.32 and $29.71 \mathrm{ppm}$ are, respectively, attributed to the $\mathrm{C}(m)$ and $\mathrm{C}(n, o)$. This result means that the aromatic and the saturated carbon in ${ }^{13} \mathrm{C}$ nuclear magnetic resonance spectra located in the $\delta_{\mathrm{C}}$ range of $110-160 \mathrm{ppm}$ and 5-55 ppm, respectively (Qiu et al. 2013). The ${ }^{1} \mathrm{H}$ nuclear magnetic resonance spectra of recycled tetrabromobisphenol A and pure tetrabromobisphenol A (Fig. 3b) show two
Fig. 2 Effect of treatment time on tetrabromobisphenol A recovery (a) and morphology of recycled tetrabromobisphenol $\mathrm{A}$ (b) and pure tetrabromobisphenol A (c). Conditions: solvent methanol; temperature $90{ }^{\circ} \mathrm{C}$; liquid to solid ratio $15: 1$. Note that the optimum treating time for tetrabromobisphenol A recovery is $120 \mathrm{~min}$. Yellow tetrabromobisphenol A powder with a purity of $95.6 \%$ is obtained, and it can be used as chemical feedstocks
Tetrabromobisphenol A Recovery (\%)
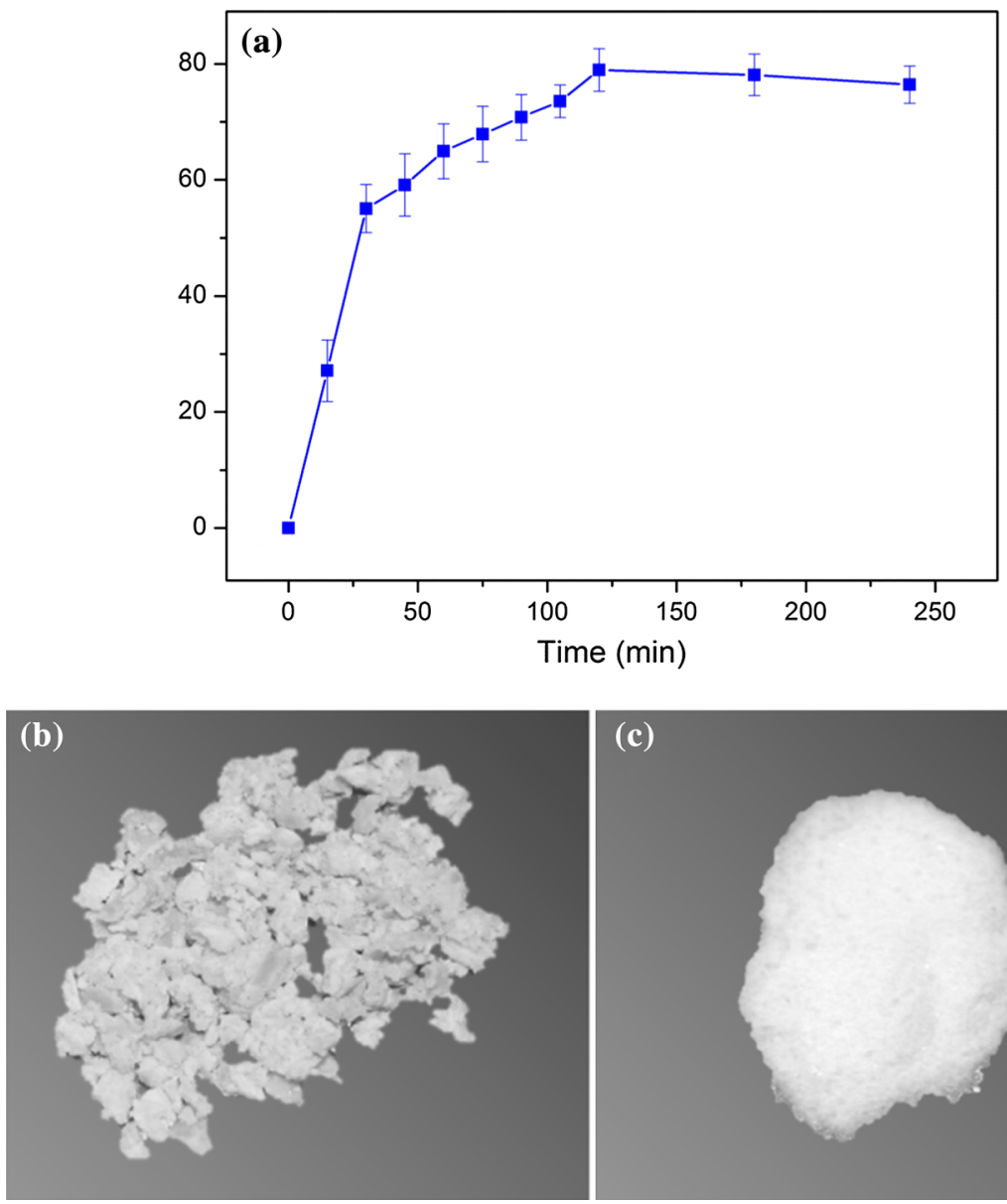

(c) 


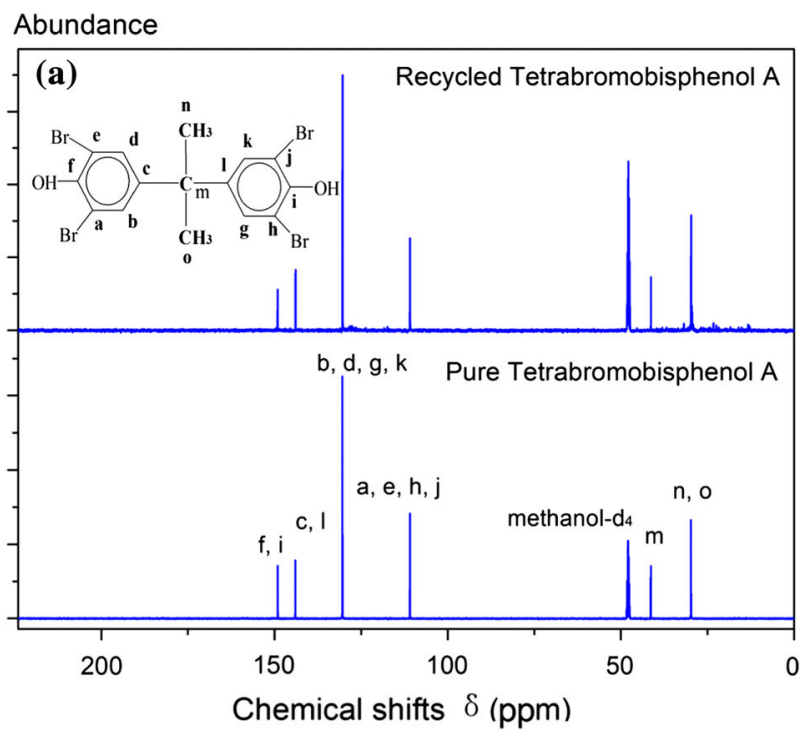

Abundance

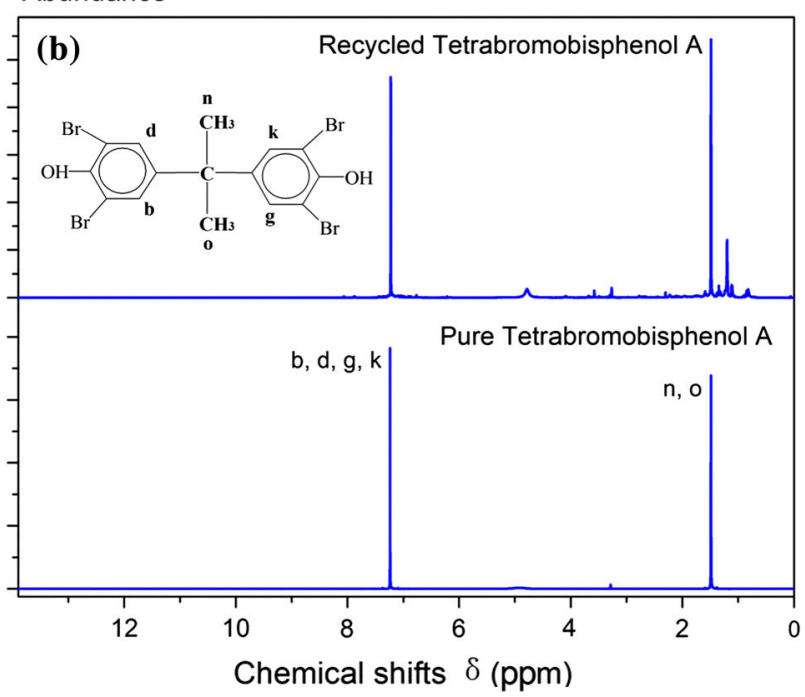

Fig. 3 Nuclear magnetic resonance spectra of the recycled tetrabromobisphenol A and pure tetrabromobisphenol A in methanol- $\mathrm{d}_{4}$ : (a) ${ }^{13} \mathrm{C}$; (b) ${ }^{1} \mathrm{H}$. Conditions: solvent methanol; temperature $90{ }^{\circ} \mathrm{C}$; time $120 \mathrm{~min}$; liquid to solid ratio $15: 1$. Note the similar ${ }^{13} \mathrm{C}$ and ${ }^{1} \mathrm{H}$ nuclear magnetic resonance profiles of the recycled tetrabromobisphenol A and tetrabromobisphenol A standard material confirm that the obtained product is typical tetrabromobisphenol A

separate signals for six protons present in tetrabromobisphenol A. The relative high chemical shift at $7.11 \mathrm{ppm}$ shows that $\mathrm{H}(b, d, g, k)$ of the aromatic benzene are identical, and the signal at $1.69 \mathrm{ppm}$ is ascribed to the $\mathrm{H}(n, o)$, which indicates that the protons on phenyl ring yield nuclear magnetic resonance signals in the $\delta_{\mathrm{H}}$ region of 6-8.5 ppm (Qiu et al. 2013). The ${ }^{13} \mathrm{C}$ and ${ }^{1} \mathrm{H}$ nuclear magnetic resonance profiles of the recycled tetrabromobisphenol $\mathrm{A}$ are in good agreement with tetrabromobisphenol A standard material, which further confirm that the obtained product is typical tetrabromobisphenol A. In order to evaluate the mechanism of tetrabromobisphenol A recovery, kinetics analysis of the solvothermal process was modeled according to shrinking core model.

\section{Solvothermal recovery mechanism}

Assuming that tetrabromobisphenol A particles is spherical shrinking core, the shrinking core model suggests that the rate of controlling steps of dissolution process are surface dissolution, diffusion across polymer layer, both interface transfer and diffusion across polymer layer (Liu et al. 2012). The corresponding shrinking core model was, respectively, expressed by Eqs. (1)-(3) as follows:

The process is controlled by surface dissolution (Liu et al. 2012):

$1-(1-\alpha)^{1 / 3}=k_{1} t$

The process is controlled by diffusion across polymer layer (Liu et al. 2012):

$1-(2 / 3) \alpha-(1-\alpha)^{2 / 3}=k_{2} t$

The process is controlled by both interface transfer and diffusion across polymer layer (Dickinson and Heal 1999):

$(1 / 3) \ln (1-\alpha)+(1-\alpha)^{-1 / 3}-1=k_{3} t$

where $\alpha$ is recovery efficiency of tetrabromobisphenol A, $k_{1}, k_{2}, k_{3}$ are rate constants and $t$ is treatment time.

It can be seen from Eqs. (1)-(3) that the plots of $1-(1-\alpha)^{1 / 3}, \quad 1-(2 / 3) \alpha-(1-\alpha)^{2 / 3}$ and $(1 / 3) \ln$ $(1-\alpha)+(1-\alpha)^{-1 / 3}-1$ versus time are straight lines under corresponding conditions. The expressions of $1-(1-\alpha)^{1 / 3}, \quad 1-(2 / 3) \alpha-(1-\alpha)^{2 / 3}$ and $(1 / 3) \ln$ $(1-\alpha)+(1-\alpha)^{-1 / 3}-1$ were thus plotted versus the treatment time in order for the kinetics analysis of solvothermal process (Fig. 4). The linear correlation coefficients were used to assess the correlation of the kinetics data with these models. By comparing the linear correlation coefficients in Fig. 4, it can be observed that Eqs. (2) and (3) fit equally well with the kinetics analysis of solvothermal process, implying that mainly diffusion across polymer layer affects the recovery rate of tetrabromobisphenol $\mathrm{A}$ in the solvothermal process.

Since additive-brominated flame retardants, including tetrabromobisphenol A, polybrominated diphenyl ethers and hexabromocyclododecane, display similar properties in electrical and electronic waste plastic, it is indisputable that solvothermal procedure own the infinite superiority in recycling all additive-brominated flame retardants contained in electrical and electronic waste plastic, and the kinetics analysis contribute to discover the rate controlling steps of these solvothermal processes. 

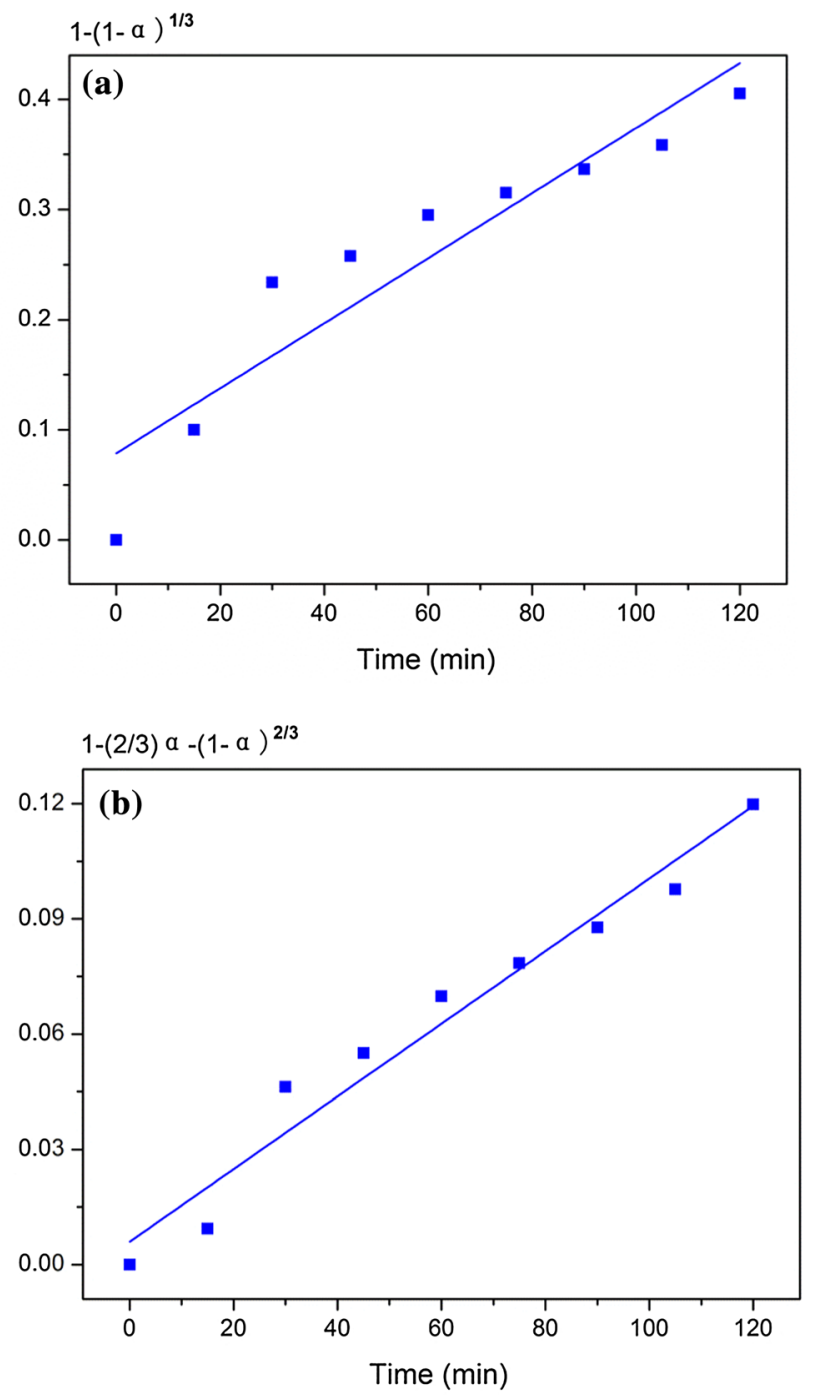

$(1 / 3) \ln (1-\alpha)+(1-\alpha)^{-1 / 3}-1$

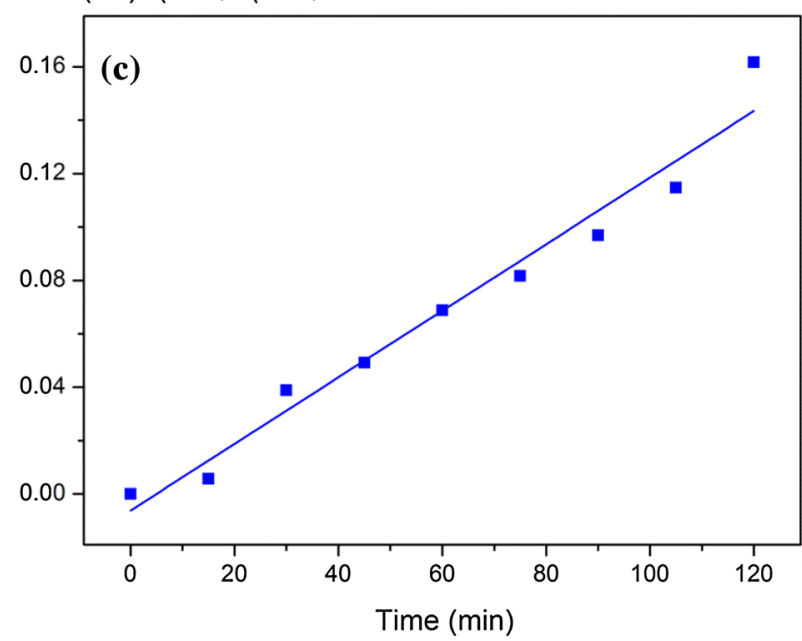

Fig. 4 Plots of $1-(1-\alpha)^{1 / 3}, 1-(2 / 3) \alpha-(1-\alpha)^{2 / 3}$ and $(1 /$ 3) $\ln (1-\alpha)+(1-\alpha)^{-1 / 3}-1$ versus time. Conditions: solvent methanol; temperature $90{ }^{\circ} \mathrm{C}$; liquid to solid ratio $15: 1$. Note that the correlation coefficients for $1-(1-\alpha)^{1 / 3}, 1-(2 / 3) \alpha-(1-$ $\alpha)^{2 / 3}$ and $(1 / 3) \ln (1-\alpha)+(1-\alpha)^{-1 / 3}-1$ are $0.86,0.96$ and 0.96 , respectively, implying that mainly diffusion across polymer layer affects the recovery rate of tetrabromobisphenol $\mathrm{A}$ in the solvothermal process

\section{Conclusion}

Solvothermal process was an efficient and practical technique for recovery of additive tetrabromobisphenol A from waste computer housing plastic. High tetrabromobisphenol A recovery efficiency of $78.9 \%$ with a purity of $95.6 \%$ was achieved by this technique in this study. The structure of the recycled tetrabromobisphenol $\mathrm{A}$ was in good agreement with tetrabromobisphenol A standard material. Kinetics analysis indicated that tetrabromobisphenol A recovery rate was mainly controlled by diffusion across polymer layer in the solvothermal process. Solvothermal process is an important alternative to conventional disposal technologies in point of non-secondary pollution. It is promising for all additive-brominated flame retardants recovery from electrical and electronic waste plastic.

Acknowledgments The authors are grateful for financial supports from the National Natural Science Foundation of China (21077120, 51278485), the China Post-doctoral Science Foundation (2013M530750) and the Science Promotion Program of Research Center for Eco-Environmental Sciences, Chinese Academy of Sciences (YSW2013B05).

\section{References}

Alaee M, Arias P, Sjödin A, Bergman $\AA$ (2003) An overview of commercially used brominated flame retardants, their applications, their use patterns in different countries/regions and possible modes of release. Environ Int 29:683-689

Altwaiq AM, Wolf M, van Eldik R (2003) Extraction of brominated flame retardants from polymeric waste material using different solvents and supercritical carbon dioxide. Anal Chim Acta 491(1):111-123

Dickinson C, Heal G (1999) Solid-liquid diffusion controlled rate equations. Thermochim Acta 340:89-103

Gamse T, Steinkellner F, Marr R, Alessi P, Kikic I (2000) Solubility studies of organic flame retardants in supercritical $\mathrm{CO}_{2}$. Ind Eng Chem Res 39(12):4888-4890

Guo JY, Guo J, Xu ZM (2009) Recycling of non-metallic fractions from waste printed circuit boards: a review. J Hazard Mater 168(2-3):567-590

Heart S (2008) Environmental impacts and use of brominated flame retardants in electrical and electronic equipment. Environmentalist 28(4):348-357 
Imai T, Hamm S, Rothenbacher KP (2003) Comparison of the recyclability of flame-retarded plastics. Environ Sci Technol 37(3):652-656

Li Y, Wang T, Hashi Y, Li H, Lin JM (2009) Determination of brominated flame retardants in electrical and electronic equipments with microwave-assisted extraction and gas chromatography-mass spectrometry. Talanta 78(4):1429-1435

Liu GB, Dai L, Gao X, Li MK, Thiemann T (2006) Reductive degradation of tetrabromobisphenol A (TBBPA) in aqueous medium. Green Chem 8:781-783

Liu ZX, Yin ZL, Hu HP, Chen QY (2012) Leaching kinetics of lowgrade copper ore containing calcium-magnesium carbonate in ammonia-ammonium sulfate solution with persulfate. T Nonferr Metal Soc 22(11):2822-2830

Luo S, Yang S, Sun C, Wang X (2011) Feasibility of a two-stage reduction/subsequent oxidation for treating tetrabromobisphenol A in aqueous solutions. Water Res 45:1519-1528

Osako M, Kim YJ, Sakai SI (2004) Leaching of brominated flame retardants in leachate from landfills in Japan. Chemosphere 57(10): 1571-1579

Qiu S, Wei J, Pan F, Liu J, Zhang A (2013) Vibrational, NMR

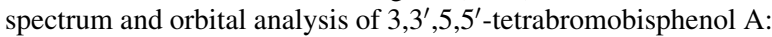
a combined experimental and computational study. Spectrochim Acta A 106:38-44

Sakai S, Watanabe J, Honda Y, Takatsuki H, Aoki I, Futamatsu M, Shiozaki K (2001) Combustion of brominated flame retardants and behavior of its byproducts. Chemosphere 42(5-7):519-531
Schlummer M, Brandl F, Mäurer A, van Eldik R (2005) Analysis of flame retardant additives in polymer fractions of waste of electric and electronic equipment (WEEE) by means of HPLC-UV/MS and GPC-HPLC-UV. J Chromatogr A 1064(1):39-51

Schlummer M, Gruber L, Mäurer A, Wolz G, van Eldik R (2007) Characterisation of polymer fractions from waste electrical and electronic equipment (WEEE) and implications for waste management. Chemosphere 67(9):1866-1876

Vilaplana F, Karlsson P, Ribes-Greus A, Ivarsson P, Karlsson S (2008) Analysis of brominated flame retardants in styrenic polymers: comparison of the extraction efficiency of ultrasonication, microwave-assisted extraction and pressurised liquid extraction. J Chromatogr A 1196:139-146

Vilaplana F, Ribes-Greus A, Karlsson S (2009) Microwave-assisted extraction for qualitative and quantitative determination of brominated flame retardants in styrenic plastic fractions from waste electrical and electronic equipment (WEEE). Talanta 78(1):33-39

Wang H, Hirahara M, Goto M, Hirose T (2004) Extraction of flame retardants from electronic printed circuit board by supercritical carbon dioxide. J Supercrit Fluid 29:251-256

Zhang CC, Zhang FS (2012) Removal of brominated flame retardant from electrical and electronic waste plastic by solvothermal technique. J Hazard Mater 221:193-198 